\title{
Efeito de Estratégias de Codificação sobre a Memória Contextual em Idosos
}

\author{
Effect of Encoding Strategies on Contextual Memory in Elders
}

\author{
Rosane Papaleo Freire ${ }^{a}$, Joana Bisol Balardin $^{a}$, Fábio Caldana $^{a}$, Cristiane Moro dos Santos $^{a}$, \\ Luciana Cunha Krebs ${ }^{a}$, Valdemarina Bidone de Azevedo e Souza* ${ }^{*}$, \\ Nadja Schröder ${ }^{a} \&$ Elke Bromberg ${ }^{* * a}$ \\ ${ }^{a}$ Pontifícia Universidade Católica do Rio Grande do Sul \\ ${ }^{b}$ Faculdades Metodistas de Porto Alegre
}

\begin{abstract}
Resumo
Estudos recentes mostram que a memória contextual parece ser especialmente suscetível aos efeitos negativos do envelhecimento sobre a cognição. O objetivo deste estudo foi investigar o efeito do uso de estratégias de codificação no desempenho de idosos em uma tarefa de memória contextual. Vinte e quatro idosos e vinte e um jovens foram divididos em dois subgrupos para a realização da tarefa: um que recebeu orientação específica para estabelecimento do vínculo item-contexto e outro que não recebeu essa orientação na fase de codificação. $\mathrm{Na}$ fase de teste, os participantes foram submetidos às tarefas de reconhecimento do objeto e do contexto. Os resultados indicam que a estratégia de estabelecimento do vínculo item-contexto foi capaz de reverter os déficits de memória contextual dos idosos.

Palavras-chave: Memória; envelhecimento; cognição.
\end{abstract}

\begin{abstract}
Previous researches suggest that contextual memory is especially susceptible to the negative effects of aging upon cognition. The purpose of this study was to evaluate the effects of memorization strategies on the performance of twenty-four elders and twenty-one young participants on contextual memory task. Within each of the age groups, the participants were divided into those that received or did not receive specific orientation to link objects to a context. At test session, participants were engaged in object and context recognition tests. Findings showed that the specific orientation to link object to context was able to revert the contextual memory deficits of the elders.

Keywords: Memory; aging; cognition.
\end{abstract}

Queixas de memória são comuns em uma grande parcela das pessoas com mais de 60 anos. Apesar de freqüentes, os déficits de memória associados ao envelhecimento não são evidenciados em todos os sistemas de memória de forma igualitária. Sabe-se que as alterações de memória associadas à idade são mais pronunciadas em tarefas de memória de trabalho e de memória episódica que requerem evocação livre e a recordação do contexto no qual o evento/ informação ocorreu (Grady \& Craik, 2000). Embora os idosos saibam que um determinado evento ocorreu ou tenham conhecimento de um fato específico, eles são menos hábeis em recordar onde ou quando o evento aconteceu, como adquiriram o conhecimento sobre ele e quais as características ou atributos da situação de aprendizado. Esse tipo de memória, que parece ser especialmente suscetível aos efeitos negativos do envelhecimento, tem sido referido

\footnotetext{
* In Memoriam.

*** Endereço para correspondência: Av. Ipiranga, 6681, Prédio

12, Bloco A, $2^{\circ}$ andar, Porto Alegre, RS, 90619-900. Tel.: 3320

3500; Ramal 4743.E-mail: bromberg@ pucrs.br
}

como memória contextual (Johnson, Hashtroudi \& Lindsay, 1993).

Os efeitos negativos do envelhecimento sobre a memória contextual vêm sendo relatados por inúmeros estudos que utilizam diferentes tipos de contextos, como o temporal (Fabiani \& Friedman, 1997; Trott, Friedman, Ritter, Fabiani \& Snodgrass, 1999), o espacial (Cherry \& Jones, 1999), e o perceptivo (Rahhal, May \& Hasher, 2002), os quais, de forma geral, requerem a realização de julgamentos em relação à quando, onde e por qual via sensorial os itens foram adquiridos. Uma importante constatação desses estudos é que, ao contrário do que ocorre com a memória contextual, o simples reconhecimento dos itens-alvo, ou seja, reconhecer se o item foi visto previamente ou não, é um processo que parece não ser afetado pelo envelhecimento. A observação dessa dissociação tem gerado um amplo debate sobre as bases neurobiológicas e cognitivas destes dois tipos de memória, assim como tem contribuído para a discussão a respeito das estruturas subjacentes ao estabelecimento da memória contextual. Alguns estudos 
referem que as estruturas do lobo temporal medial seriam as responsáveis pelos processos da memória contextual (Davachi, Mitchell \& Wagner, 2003; Gold et al, 2006). Entretanto, trabalhos com idosos têm atribuído ao lobo frontal, e às suas alterações funcionais e estruturais relacionadas ao envelhecimento, o principal papel nos déficits de memória contextual, uma vez que as operações necessárias para a realização do vínculo entre o item e o contexto parecem ser suportadas principalmente pelo córtex pré-frontal (Glisky, Rubin \& Davidson 2001; Wegesin, Jacobs, Zubin, Ventura \& Stern, 2000).

Além da caracterização das alterações de memória contextual apresentadas pelos idosos, a possibilidade de reversão de tais déficits tem constituído um interessante e promissor campo de pesquisa nas neurociências. Diferentes estudos têm sugerido que no processo de envelhecimento, na ausência de patologias específicas que comprometam a atividade do sistema nervoso, os déficits cognitivos relacionados ao lobo frontal podem não ser resultado de uma perda irreversível das funções deste sistema. Evidências empíricas mostram que esses déficits podem ser amenizados, ou até mesmo revertidos, em situações de teste nas quais os idosos são encorajados a fazer uso de todos os recursos estratégicos disponíveis (Dywan, Segalowitz, Webster, Hendry \& Harding, 2001; Glisky et al., 2001; Logan, Sanders, Snyder, Morris \& Buckner, 2002). Esses achados advêm, em grande parte, de tarefas nas quais a memorização é intencional. Entretanto, a maioria das memórias é adquirida de forma incidental, ou seja, sem que se tenha qualquer intenção de recordá-las posteriormente.

O presente trabalho investiga o desempenho de idosos em uma tarefa de memória contextual incidental e busca verificar o efeito do uso de estratégias específicas na fase de aquisição da memória no desempenho desses sujeitos em relação a indivíduos jovens. Como hipótese, acreditase que os déficits de memória contextual apresentados pelos idosos estejam relacionados à inabilidade de realizar espontaneamente o vínculo entre item e contexto, e que estratégias que visem induzir essa combinação podem reverter tais déficits. A importância da investigação e da constatação da reversão de possíveis déficits cognitivos associados ao envelhecimento, sustentada nas abordagens que priorizam os ganhos ocorridos nessa etapa do ciclo vital, reside principalmente na possibilidade de criar formas de intervenção e de adaptação das capacidades cognitivas dos idosos às demandas sociais e ambientais (Parente \& Wagner, 2006).

\section{Método}

\section{Participantes}

Participaram deste estudo 24 indivíduos idosos, na faixa etária entre 60 e 75 anos, e 21 jovens, entre 20 e 25 anos. Os idosos foram alocados em grupos de convivência da cidade de Porto Alegre (RS), cadastrados no Conselho Municipal do Idoso, e os jovens entre participantes do programa Educação de Jovens e Adultos (EJA) e entre universi- tários de uma instituição de ensino superior particular da cidade de Porto Alegre (RS).

Todos os participantes foram submetidos a um questionário de identificação contendo questões sobre saúde e hábitos de vida, e a instrumentos de rastreio de depressão (Escala de Depressão Geriátrica - GDS, Yesavage et al., 1983, e Mini International Neuropsychiatric Interview MINI, Sheehan et al., 1998), de déficits cognitivos (Mini Exame do Estado Mental - MEEM, Folstein, Folstein \& McHugh, 1975) e ao subteste de vocabulário da Escala Weschler de Inteligência para Adultos (Nascimento, 2000). O subteste vocabulário foi utilizado como uma medida de inteligência geral com o objetivo de assegurar-se que os participantes dos diferentes grupos experimentais tivessem habilidades semelhantes para compreensão e realização das tarefas de memória. Foram excluídos jovens e idosos com déficits sensoriais visuais e auditivos incompatíveis com a realização das tarefas e com alterações neurológicas autoreferidas (ex. traumatismos, história prévia de acidente vascular cerebral, epilepsia, patologias neurodegenerativas como Doença de Parkinson), além de pontuação no MEEM compatível com déficits cognitivos e na GDS e MINI compatível com depressão. Também foram excluídos indivíduos que relataram terem feito uso de antidepressivos nos seis meses antecedentes a participação no estudo, e aqueles com utilização prévia ou atual de fármacos psicoativos (ex. anticonvulsionantes e benzodiazepínicos) e de drogas de abuso (ex. álcool). Os voluntários foram divididos em quatro grupos experimentais, de acordo com a idade (jovens e idosos) e o tipo de instrução recebida para a realização da tarefa de memória contextual (sem orientação específica para estabelecimento do vínculo entre o objeto e o contexto e com orientação específica para estabelecimento do vínculo entre o objeto e o contexto). Todos os participantes assinaram um termo de consentimento livre e esclarecido e não receberam qualquer tipo de remuneração e/ou auxílio para participar do estudo. O projeto referente a presente pesquisa obteve aprovação do Comitê de Ética em Pesquisa da Pontifícia Universidade Católica do Rio Grande do Sul (CEP-PUCRS 007/03).

As características demográficas dos participantes são descritas na Tabela 1. A análise de variância (ANOVA) não indicou diferença significativa entre os quatro grupos experimentais em relação às variáveis escolaridade, escores no subteste vocabulário e no $\operatorname{MEEM}(p>0,05)$.

\section{Materiais}

O material das tarefas de memória foi composto por uma série de diapositivos retratando 44 objetos de diversas categorias semânticas (ex. ferramentas, brinquedos, utensílios domésticos, peças do vestuário, material de higiene), situados em dois ambientes distintos, uma sala de visitas e um escritório. A escolha dos objetos que compuseram os diapositivos priorizou aqueles que apresentavam o mesmo grau de relação semântica com os dois ambientes nos quais foram retratados, ou seja, objetos que normalmente não seriam encontrados nem na sala nem no escritório (cate- 
Tabela 1

Características Demográficas e Cognitivas dos Grupos Experimentais

\begin{tabular}{lcccc}
\hline \multicolumn{2}{c}{ Idosos } & \multicolumn{2}{c}{ Jovens } \\
\hline & Sem orientação $(n=12)$ & Com orientação $(n=12)$ & Sem orientação $(n=11)$ & Com orientação $(n=10)$ \\
\hline Idade & $69,08 \pm 1,72$ & $67,08 \pm 1,46$ & $22,09 \pm 0,49$ & $22,70 \pm 053$ \\
Escolaridade & $8,00 \pm 1,6$ & $8,00 \pm 1,65$ & $6,64 \pm 0,88$ & $7,00 \pm 1,38$ \\
Vocabulário $^{\text {a }}$ & $9,00 \pm 0,89$ & $9,58 \pm 0,90$ & $8,73 \pm 0,46$ & $9,00 \pm 0,66$ \\
MEEM & $27,00 \pm 0,98$ & $24,41 \pm 1,29$ & $25,54 \pm 0,63$ & $26,40 \pm 0,54$ \\
\hline
\end{tabular}

Nota . MEEM = Mini Exame do Estado Mental. ${ }^{a}$ Escores da Escala Wechsler de Inteligência para Adultos (Nascimento, 2000).

goria A) ou objetos que teriam a mesma probabilidade de serem encontrados na sala ou no escritório (categoria B). Este conjunto de 88 diapositivos, retratando 44 objetos em dois ambientes distintos, foi utilizado tanto para a tarefa de memória de reconhecimento do objeto quanto para a tarefa de memória contextual. Os diapositivos a serem utilizados em uma ou outra tarefa foram escolhidos aleatoriamente para cada sujeito, tomando-se o cuidado de não reutilizar diapositivos já apresentados na tarefa de reconhecimento do objeto para a tarefa de memória contextual. Adicionalmente, foram utilizados vários critérios, abaixo descritos, na composição do conjunto de diapositivos a serem apresentados nas sessões de estudo e teste de cada tarefa, para garantir a obtenção de uma amostragem homogênea.

Para a sessão de estudo da tarefa de memória de reconhecimento do objeto, foram selecionados aleatoriamente, para cada participante, 16 diapositivos de objetos (contrabalanceados para as categorias A e B) igualmente distribuídos entre os dois ambientes: 8 objetos na sala de visitas e 8 no escritório. Para a sessão de teste, aos 16 diapositivos utilizados na sessão de estudo, somaram-se outros 16 diapositivos, retratando objetos diferentes daqueles da sessão de estudo e igualmente distribuídos entre os dois ambientes, que foram utilizados como distratores.

A composição do material da tarefa de memória contextual seguiu os mesmos critérios adotados para a seleção do material da tarefa de memória de reconhecimento do objeto, exceto pelo número de diapositivos utilizados nas sessões de estudo e teste. Para a tarefa de memória contextual foram selecionados 12 diapositivos para a sessão de estudo e 24 para a sessão de teste. Este ajuste foi realizado simplesmente para aumentar a margem de segurança na interpretação dos dados da tarefa de memória contextual, garantindo que os resultados não seriam decorrentes de alguma interferência relacionada à dificuldade de reconhecimento dos objetos (já que na tarefa de memória de reconhecimento do objeto, utilizando-se 16 slides na fase de estudo, jovens e idosos apresentaram desempenho semelhante). Portanto, na sessão de estudo da tarefa de memória contextual foram apresentados, para cada participante, 12 diapositivos de objetos igualmente distribuí- dos entre os dois ambientes: 6 na sala e 6 no escritório. $\mathrm{Na}$ sessão de teste foram reapresentados os 12 diapositivos utilizados na sessão de estudo, sendo que cada um deles foi pareado com o diapositivo que retratava o objeto em um ambiente diferente daquele da sessão de estudo. Portanto, se na fase de estudo o objeto aparecia na sala, na fase de teste eram mostrados, consecutivamente, os diapositivos retratando o objeto na sala e no escritório. A ordem de apresentação dos objetos na fase de estudo e teste foi estabelecida aleatoriamente, assim como a ordem na qual eram mostrados os slides pareados do teste que apresentavam consecutivamente o mesmo objeto nos dois ambientes distintos. Os diapositivos foram projetados utilizando-se um projetor Kodak Ektagraphic, resultando em uma imagem de 100 $\mathrm{x} 130 \mathrm{~cm}$.

\section{Procedimentos}

Para a realização das tarefas de memória, duas sessões de estudo-teste foram realizadas com cada sujeito. A primeira delas referiu-se à tarefa de memória de reconhecimento do objeto e a segunda à tarefa de memória contextual. Na primeira sessão de estudo dos grupos experimentais, referente à tarefa de memória de reconhecimento do objeto, foram apresentados 16 diapositivos de objetos (igualmente divididos entre os dois ambientes), e os participantes foram instruídos a realizar um julgamento sobre a freqüência com que utilizavam cada um dos objetos no seu dia-a-dia, tendo como alternativas as respostas nunca, às vezes ou sempre. Após 3 minutos de intervalo, no qual foi realizada uma tarefa distratora (repetição de dígitos na ordem direta e reversa), realizou-se a sessão de teste, quando foram apresentados 32 diapositivos: 16 com os mesmos objetos já apresentados na fase de estudo e 16 com objetos novos, metade em cada ambiente. Os participantes, então, realizaram um teste de memória de reconhecimento sobre os objetos, sendo solicitados a identificar quais objetos eram novos e quais já haviam sido visualizados na sessão de estudo. Após um intervalo de 10 minutos, os participantes realizaram a tarefa de memória contextual. Metade dos voluntários dos grupos de jovens e de idosos foi submetida a uma sessão de estudo idêntica àquela da tarefa da memória de reconhecimento do objeto, sendo solicitada a reali- 
zar o julgamento sobre a freqüência de uso do objeto no seu dia-a-dia, porém com a exibição de apenas 12 diapositivos. O tipo de julgamento realizado pelos participantes teve como objetivo orientar sua atenção especificamente para o objeto. Para esses grupos experimentais, na sessão de teste da tarefa de memória contextual, foram apresentados 24 diapositivos: cada um dos 12 objetos que tinham aparecido durante a fase de estudo foi reapresentado seqüencialmente nos dois ambientes (sala e escritório). Cada diapositivo foi exibido durante 3 segundos. Os participantes, então, foram solicitados a indicar, para cada um dos objetos, em qual ambiente (sala de visitas ou escritório) o mesmo tinha aparecido na fase de estudo, ou seja, realizaram uma tarefa de memória contextual.

A outra metade dos participantes dos grupos de jovens e idosos também foi submetida à tarefa de memória contextual. Entretanto, durante a sessão de estudo, os voluntários realizaram outro tipo de julgamento em relação ao objeto, sendo solicitados a indicar se o objeto era adequado ou não ao ambiente no qual estava inserido. O tipo de julgamento realizado pelos participantes teve como objetivo orientar sua atenção também para o ambiente no qual o objeto estava inserido. A fase de teste da tarefa foi semelhante àquela dos outros grupos experimentais. Não houve limite de tempo para resposta em nenhuma das tarefas, seja na fase de estudo ou de teste, para todos os grupos experimentais. Durante a fase de estudo das duas tarefas realizadas, os voluntários não foram informados de que seriam testados em relação à memória de reconhecimento do objeto apresentado ou ao contexto no qual o mesmo estava inserido, caracterizando um procedimento incidental de investigação da memória. A ordem de apresentação das tarefas de memória, assim como dos outros instrumentos de caracterização da amostra, foi a mesma para todos os participantes.

\section{Análise Estatística}

Os resultados das tarefas de memória de reconhecimento do objeto e de memória contextual foram submetidos à análise de variância (ANOVA) de dois fatores, utilizando a idade (jovens, adultos) como fator inter-grupos, e o tipo de instrução recebida para a realização das tarefas (com estratégia, sem estratégia) como fator intra-grupos. Quando necessário, a ANOVA de dois fatores foi seguida do teste post hoc de Tukey. Valores de $p<0,05$ foram utilizados como indicativo de significância estatística. Os resultados serão apresentados como porcentagem de acertos e expressos como média_erro padrão (EP).

\section{Resultados}

A Figura 1 apresenta as porcentagens de acertos nas tarefas de reconhecimento do objeto e de memória contextual. $\mathrm{Na}$ tarefa de reconhecimento do objeto, a ANOVA de dois fatores não indicou efeitos da idade $(F<1)$, do tipo de instrução recebida $(F<1)$, e interação entre o tipo de instrução e a idade $(F<1)$. Na tarefa de memória contextual, a ANOVA de dois fatores indicou um efeito significativo da idade
$\left(F_{(1,41)}=13,88 ; p<0,001\right)$, do tipo de instrução recebida $\left(F_{(1,41)}=8,84 ; p<0,001\right)$ e uma interação entre esses dois fatores $\left(F_{(1,41)}=3,85 ; p<0,05\right)$. Os efeitos significativos da idade e do tipo de instrução indicam que os escores do grupo de jovens $(85,92 \pm 3,58)$ foram significativamente maiores em relação aos idosos $(67,62 \pm 3,35)$, e que a instrução que encorajou o estabelecimento do vínculo entre o item e o contexto ocasionou um melhor desempenho $(84,07 \pm 3,51) \mathrm{em}$ relação à condição na qual o vínculo não foi encorajado $(69,47 \pm 3,42)$. A interação entre os fatores idade e tipo de instrução revela que o uso da pista para o estabelecimento do vínculo entre o item e o contexto melhorou de forma significativa apenas o desempenho dos idosos, uma vez que os jovens não apresentaram diferenças significativas entre as duas condições de avaliação. $\mathrm{O}$ teste post hoc de Tukey indicou diferença significativa $(p<0,05)$ apenas entre o grupo de idosos submetidos à tarefa de memória contextual sem orientação específica para o estabelecimento do vínculo entre o objeto e o contexto $(55,5 \pm 4,74)$ e os demais grupos experimentais (idosos com orientação $79,75 \pm 4,74$; adultos com orientação $88,4 \pm 5,19$ e adultos sem orientação $83,45 \pm 4,95)$.

\section{A}

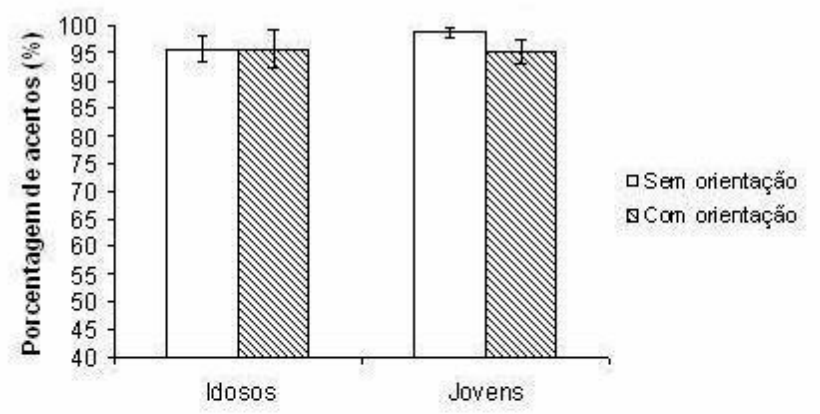

B

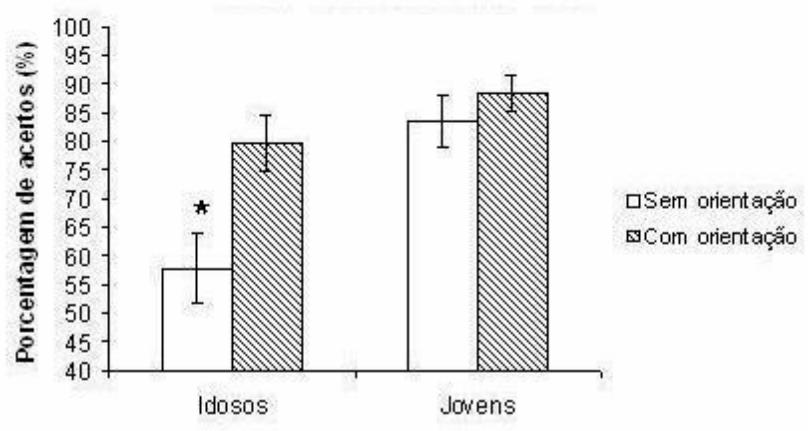

Figura 1. Porcentagem de acertos de idosos e jovens submetidos às tarefas de reconhecimento do objeto (a) e de memória contextual (b).

Nota. Porcentagem de acertos expressa como média_EP. *Diferença significativa em relação aos demais grupos experimentais na tarefa de memória contextual. Teste de Tukey, $p<0,05$. 


\section{Discussão}

Os resultados do presente estudo demonstram que os idosos apresentam déficits de memória contextual que podem ser revertidos pelo uso de estratégias na fase de aquisição da memória. O desempenho dos idosos foi semelhante ao dos jovens apenas na situação na qual o foco da atenção dos idosos foi orientado para o vínculo entre o contexto (ambiente) e o item (objeto), sendo que o desempenho dos jovens foi semelhante nas duas condições de avaliação, com e sem o uso da estratégia de codificação. Já na tarefa de memória de reconhecimento do objeto, os idosos tiveram desempenho semelhante ao dos jovens.

Os resultados do nosso estudo concordam com evidências prévias de que a memória para o contexto parece ser mais prejudicada pelo envelhecimento do que a memória de reconhecimento do item (Fabiani \& Friedman, 1997; Rahhal et al., 2002; Trott, Friedman, Ritter \& Fabiani, 1997), contribuindo para a hipótese de que o envelhecimento possui efeitos dissociados sobre os diferentes tipos de memória (Grady \& Craik, 2000). Nossos achados também indicam a possibilidade de que a memória para o item e a memória para o contexto representem, pelos menos parcialmente, processos ou sistemas distintos, como sugerido por Zacks, Hasher e Li (2000), uma vez que apenas na tarefa de memória contextual o desempenho dos idosos foi inferior ao dos jovens.

Apesar de ser uma questão de debate entre os pesquisadores, uma das possíveis explicações para os déficits de memória contextual associados ao envelhecimento recai sobre alterações nos mecanismos envolvidos na síntese de diferentes atributos para a formação de memórias complexas e nos processos envolvidos no acesso e na avaliação desses atributos contextuais das memórias (Siedlecki, Salthouse \& Berish, 2005). Os achados do presente estudo dão suporte a esta hipótese, uma vez que os idosos só conseguiram obter desempenho semelhante ao dos jovens na tarefa de memória contextual na condição em que foram induzidos a realizar o vínculo entre o item e o contexto, mostrando dificuldade em realizá-lo espontaneamente. Estudos têm atribuído os processos estratégicos e de varredura que o sujeito realiza para fazer o vínculo entre o item e o contexto nas fases de aquisição e evocação da memória à atividade de regiões do lobo frontal (Buckner \& Wheeler, 2001). Um importante trabalho (Glisky, Polster \& Routhieaux, 1995) mostrou uma correlação positiva entre os desempenhos de participantes idosos em testes que avaliam o funcionamento das estruturas do lobo temporal medial (LTM) e um teste de memória de reconhecimento de itens, enquanto que idosos com alto funcionamento do lobo frontal apresentaram bom desempenho em um teste de memória de reconhecimento do contexto, sugerindo que a memória para itens dependa primordialmente das estruturas do LTM e a memória do contexto seja suportada pelo lobo frontal, o que foi confirmado e ampliado por achados de estudos subseqüentes (Glisky et al., 2001; Johnson, Kounios \& Nolde, 1997). Entretanto, recentes evidências de estudos de neuroimagem mostram que a ativação da região hipocampal, do córtex peririnal e do córtex parahipocampal durante a fase de codificação da memória foi preditiva de sucesso no reconhecimento tanto do item quando do contexto em voluntários jovens saudáveis, e que pacientes com lesões restritas à região hipocampal apresentaram déficits em ambas as tarefas, sugerindo que o reconhecimento do item e do contexto parecem depender, em igual magnitude, das estruturas do LTM (Gold et al., 2006).

Do exposto acima, e considerando que os quatro grupos experimentais do nosso estudo apresentaram desempenho semelhante na tarefa de memória de reconhecimento do objeto, acreditamos que os déficits de memória contextual dos idosos do presente trabalho não possam ser atribuídos a prejuízos no reconhecimento do material da tarefa, mas que, muito provavelmente, estejam relacionados à ineficiência no uso dos processos estratégicos suportados pelo lobo frontal para unir o item ao contexto. Tais considerações são suportadas, em primeiro lugar, por teorias sobre envelhecimento cognitivo que priorizam o declínio das funções do lobo frontal em primazia a funções suportadas por outras regiões cerebrais, decorrentes de mudanças estruturais e funcionais específicas associadas ao processo de envelhecimento (Buckner et al., 2006). Na condição de codificação na qual foi estabelecido o vínculo entre o item e o contexto, isto é, quando houve facilitação do processo de síntese dos atributos da experiência para a formação de um traço de memória único, o desempenho dos idosos na tarefa de memória contextual foi semelhante ao dos jovens. Assim, nossos achados também suportam a hipótese de que, mais do que um prejuízo irreversível do sistema frontal, os déficits de memória contextual associados ao envelhecimento parecem refletir uma ineficiência de recrutamento desse sistema, que se evidencia, em estudos de neuroimagem, como uma seleção de regiões encefálicas não-específica e compensatória (Logan et al., 2002). Portanto, a estratégia utilizada no nosso estudo parece atuar como um reforço do papel executivo e de monitoramento do lobo frontal (deficitário no envelhecimento) sobre o processo de aquisição da memória contextual, papel este que vem sendo sugerido por outros estudos (Dobbins, Schacter \& Wagner, 2002; Gutchess et al., 2007; Raye, Mitchell, Nolde \& D’Esposito, 2000; Slotnick, Moo, Segal \& Hart, 2003).

Os resultados da tarefa de memória contextual também apontam claramente para o fato de que os indivíduos jovens desse estudo foram capazes de ativar espontaneamente processos de integração entre o item e o contexto, o que está de acordo com os achados de Glisky et al. (2001), uma vez que seus desempenhos foram semelhantes com e sem o uso da estratégia de codificação, ao contrário dos idosos, que foram capazes de igualar seu desempenho aos adultos apenas na condição em que o foco de sua atenção foi direcionado para o vínculo objeto-ambiente. Esses achados ampliam os resultados de trabalhos anteriores que mostram que os idosos, quando comparados aos jovens, utilizam de forma menos efetiva técnicas de memorização espontâneas, mas que podem beneficiarem-se de instruções diretas ou indiretas em relação ao uso de novas estratégias de memorização (Cavallini, Pagnin \& Vecchi, 2003; 
Connor, 2001). Uma vez que a estratégia de codificação utilizada nesse estudo foi capaz de reverter os déficits de memória contextual dos idosos saudáveis, torna-se importante considerar a implementação desta técnica em programas de treinamento e/ou oficinas de memória, e, em estudos futuros, testar o efeito das mesmas em diferentes populações com alterações de memória, como nos quadros depressivos e em doenças neurodegenerativas.

\section{Referências}

Buckner, R., Persson, J., Nyberg, L., Lind, J., Larsson, A., Nilsson, L., \& Ingvar, M. (2006). Structure-function correlates of cognitive decline in aging. Cerebral Cortex, 16(7), 907-915.

Buckner, R. L., \& Wheeler, M. E. (2001). The cognitive neuroscience of remembering. Nature Reviews Neuroscience, 2(9), 624-634.

Cavallini, E., Pagnin, A., \& Vecchi, T. (2003). Aging and everyday memory: The beneficial effect of memory training. Archives of Gerontology and Geriatrics, 37(3), 241-257.

Cherry, Q. E., \& Jones, M. W. (1999). Age-related differences in spatial memory: Effects of structural and organizational context. The Journal of General Psychology, 126(1), 53-73.

Connor, L. (2001). Memory in old age: Patterns of decline and preservation. Seminars in Speech and Language, 22(2), 117-25.

Davachi, L., Mitchell, J. P., \& Wagner, A. D. (2003). Multiple routes to memory: Distinct medial temporal lobe processes build item and source memories. Proceedings of the National Academy of Sciences of the United States of America, 100(4), 2157-2162.

Dobbins, I. G., Foley, H., Schacter, D. L., \& Wagner, A. D. (2002). Executive control during episodic retrieval: Multiple prefrontal processes subserve source memory. Neuron, 35(5), 989-996.

Dywan, J., Segalowitz, S. J., Webster, L., Hendry, K., \& Harding, J. (2001). Event-related potential evidence for age-related differences in attentional allocation during a source monitoring task. Developmental Neuropsychology, 19(1), 99-120.

Fabiani, M., \& Friedman, D. (1997). Dissociations between recency and recognition memory in aging. Neuropsychologia, 35(2), 129-141.

Folstein, M. F., Folstein, S. E., \& McHugh, P. R. (1975). "MiniMental State": A practical method for grading the cognitive state of patients for the clinician. Journal of Psychiatric Research, 12(3), 189-98.

Glisky, E. L., Polster, M. R., \& Routhieaux, B. C. (1995). Double dissociation between item and source memory. Neuropsychology, 9(2), 229-235.

Glisky, E. L., Rubin, S. R., \& Davidson, P. S. R. (2001). Source memory in older adults: An encoding or retrieval problem. Journal of Experimental Psychology: Learning, Memory and Cognition, 27(5), 1131-1146.

Gold, J. J., Smith, C. N., Bayley, P. J., Shrager, Y., Brewer, J. B., Stark, C. E., Hopkins, R. O., \& Squire, L. R. (2006). Item memory, source memory, and the medial temporal lobe: Concordant findings from fMRI and memory-impaired patients. Proceedings of the National Academy of Sciences of the United States of America, 103(24), 9351-9356.

Grady, C. L., \& Craik, F. I. (2000). Changes in memory processing with age. Current Opinion in Neurobiology, 10(2), 224-31.

Gutchess, A., Hebrank, A., Sutton, B. P., Leshikar, E., Chee, M. W. L., Tan, J. C., Goh, J. O. S., \& Park, D. C. (2007). Contextual interference in recognition memory with age. Neuroimage, 35, 1338-1347.
Johnson, M. K., Hashtroudi, S., \& Lindsay, D. S. (1993). Source monitoring. Psychological Bulletin, 114(1), 3-28.

Johnson, M. K., Kounios, J., \& Nolde, S. F. (1997). Electrophysiological brain activity and memory source monitoring. Neuroreport, 8(5), 1317-1320.

Logan, J. M., Sanders, A. L., Snyder, A. Z., Morris, J. C., \& Buckner, R. L. (2002). Under-recruitment and nonselective recruitment: Dissociable neural mechanisms associated with aging. Neuron, 33(5), 827-840.

Nascimento, E. (2000). WAIS III. In J. A. Cunha (Ed.), Psicodiagnóstico: Vol. 5 (5. ed., pp. 615-617). Porto Alegre, RS: Artes Médicas.

Parente, M. A. M. P., \& Wagner, G. P. (2006). Teorias abrangentes sobre envelhecimento cognitivo. In M. A. M. P. Parente (Ed.), Cognição e envelhecimento (pp. 32-32). Porto Alegre, RS: Artmed.

Rahhal, T. A., May, C. P., \& Hasher, L. (2002). Truth and character: Sources that older adults can remember. Psychological Science, 13(2), 101-105.

Raye, C. L., Johnson, M. K., Mitchell, K. J., Nolde, S. F., \& D'Esposito, M. (2000). fMRI investigations of left and right PFC contributions to episodic remembering. Psychobiology, 28(2), 197-206.

Sheehan, D. V., Lecrubier, Y., Sheehan, K. H., Amorin, P., Janavs, J., Weiller, E., Hergueta, T., Baker, R., \& Dunbar, G. C. (1998). The Mini-International Neuropsychiatric Interview (M.I.N.I.): The development and validation of a structured diagnostic psychiatric interview for DSM-IV and ICD-10. The Journal of Clinical Psychiatry, 59(S20), 22-33.

Siedlecki, K. L., Salthouse, T. A., \& Berish, D. E. (2005). Is there anything special about the aging of source memory? Psychology and Aging, 20(1), 19-32.

Slotnick, S. D., Moo, L. R., Segal, J. B., \& Hart, J., Jr. (2003). Distinct prefrontal cortex activity associated with item memory and source memory for visual shapes. Cognitive Brain Research, 17(1), 75-82.

Trott, C. T., Friedman, D., Ritter, W., \& Fabiani, M. (1997). Item and source memory: Differential age effects revealed by event-related potentials. Neuroreport, 8(15), 3373-3378.

Trott, C. T., Friedman, D., Ritter, W., Fabiani, M., \& Snodgrass, J. G. (1999). Episodic priming and memory for temporal source: Event-related potentials reveal age-related differences in prefrontal functioning. Psychology and Aging, 14(3), 390-413.

Wegesin D. J., Jacobs, D. M., Zubin, N. R., Ventura, P. R., \& Stern, Y. (2000). Source memory and encoding strategy in normal aging. Journal of Clinical and Experimental Neuropsychology, 22(4), 455-464.

Yesavage, J. A., Brink, T. L., Rose, T. L., Lum, O., Huang, V., Adey, M., \& Leirer, V. O. (1983). Development and validation of a geriatric depression screening scale: A preliminary report. Journal of Psychiatric Research, 17(1), 37-49.

Zacks, R. T., Hasher, L., \& Li, K. Z. H. (2000). Human memory. In O. F. T. Craik \& T. A. Salthouse (Eds.), The handbook of aging and cognition (pp. 293-357). Mahwah, NJ: Erlbaum. 